\title{
The role of the magnetic orbitals in the calculation of the magnetic coupling constants from multireference perturbation theory methods
}

\author{
Celestino Angeli ${ }^{1, a)}$ and Carmen J. Calzado ${ }^{2}$ \\ ${ }^{1}$ Dipartimento di Chimica, Università di Ferrara, Via Borsari 46, I-44121 Ferrara, Italy \\ ${ }^{2}$ Departamento de Química Física, Universidad de Sevilla, c/Profesor García González s/n, \\ E-41012 Sevilla, Spain
}

(Received 8 February 2012; accepted 21 June 2012; published online 17 July 2012)

\begin{abstract}
The use of multireference perturbation theory (MRPT) for the calculation of the magnetic coupling in binuclear complexes has shown to give poor results if applied on a minimal active space complete active space self-consistent field (CASSCF) wavefunction. In this work, we identify the origin of this problem in the starting CASSCF orbitals, which are exceedingly localized on the metal atoms. Focusing on the case of antiferromagnetic systems, it is shown that the form of the active orbitals has a dramatic effect on the relative description of the neutral and ionic structures. Finally, a simple and computational inexpensive strategy is proposed for the calculation of a set of magnetic orbitals describing in a more balanced way the neutral and ionic structures. The use of these orbitals, instead the CASSCF ones, in minimal active space MRPT2 calculations leads to a marked improvement of the $J$ values, which become in reasonable agreement with those obtained with the expensive high level difference dedicated configuration interaction approach and with the experimental values.

(C) 2012 American Institute of Physics. [http://dx.doi.org/10.1063/1.4735018]
\end{abstract}

\section{INTRODUCTION}

Many efforts have been dedicated to improve the accuracy of the theoretical evaluation of the magnetic coupling constant $(J)$ for magnetic systems, as a way of controlling and understanding the main mechanisms governing the interaction between the spin moments. Different applications have shown that the difference dedicated configuration interaction ${ }^{1-3}$ (DDCI) method, applied on the minimal complete active space configuration interaction (CASCI) wavefunctions (all magnetic orbitals and electrons are active), gives, in general, $J$ values in good agreement with the experimental finding for binuclear complexes. ${ }^{4}$ In these studies, it has been found that different starting molecular orbitals (MO), that is, state specific, triplet or singlet complete active space self consistent field (CASSCF) MOs or iterated natural MOs, give in many cases almost identical DDCI $J$ values for antiferromagnetically coupled centers. However, for some antiferromagnetic systems, a more strong dependence on the orbitals of the DDCI $J$ values has been observed, and the situation for ferromagnetic systems is even more complex, with a marked influence of the starting MOs on $J$. A strong point of the DDCI approach is that, being a wavefunction based method, it allows for a deep analysis of the physical contribution to $J$ of the different excitation classes, ${ }^{5-7}$ that is, of the different excitation processes (ligand to metal charge transfer, LMCT, metal to ligand charge transfer, MLCT, etc.). For these reasons, the DDCI studies are considered as references in this field.

Nevertheless, DDCI is computationally rather expensive, in particular, for systems with large ligands, many magnetic

\footnotetext{
a) Author to whom correspondence should be addressed. Electronic mail: anc@unife.it.
}

centers, and/or many unpaired electrons per magnetic center. Several promising strategies have been recently developed with the goal to make dealing with these large systems possible, such as the excitation selected configuration interaction $^{8}$ (EXSCI) method, but they still present quite large computational costs. In this context, a less expensive and yet reliable computational strategy is highly desirable. To this aim, multireference perturbation theory (MRPT) applied to second order for the energy appears as a natural candidate.

Many studies concerning the calculation of $J$ with MRPT2 have been published in the past, using different computational strategies. Obviously, each approach shows strong points and weaknesses, but some common considerations can be reported:

- Particular care must be used in choosing the MRPT2, with a particular attention to the definition of $\hat{\mathcal{H}}^{(0)}$ and to the possible appearance of the intruder state problem.

- In the CASPT2 (Ref. 9) (one of the most used version of MRPT2) studies, state-specific CASSCF orbitals are often used. The use of a common set of orbitals for all states (differing by the spin coupling of the magnetic electrons) gives similar results (with a slight improvement of the quality).

- In general, the MRPT2 approach only takes into account a fraction $(40 \%-70 \%$, or even less) of the effect of single and double excitations on the minimal CAS (two electrons in two MOs for binuclear $\mathrm{Cu}$ complexes).

- The use of larger active spaces (including ligand orbitals and a second set of magnetic orbitals) largely improves the MRPT2 results. 
From these considerations, one can conclude that the use of active spaces larger than the minimal space is the correct computational strategy for the calculation of $J$ at the MRPT2 level. This is actually the standard approach considered in most of the CASPT2 studies (see Ref. 10) and has received its theoretical justification in the request to improve, with respect to the minimal active space case, the amount of electron correlation treated at the zero order. Nevertheless, this approach is visibly in contrast with the final aim, that is, the identification of an effective method applicable to systems with many magnetic centers or many magnetic electrons. Indeed, in such systems an enlargement of the minimal active space makes the CASSCF step impracticable, thus preventing the application of the subsequent MRPT2 approach. For these reasons, in the past years, we have tried to understand why MRPT2 on the minimal CAS considers only a fraction of the full effect of single and double excitations. Our focus has been in particular on the $n$-electron valence state perturbation theory ${ }^{11-14}$ (NEVPT2) approach. In the following, we first report some attempts to investigate this problem for the case of antiferromagnetic systems, then we propose an explanation of its origin, and, finally, a possible computational strategy to overcome it is described and applied to a set of test binuclear $\mathrm{Cu}$ complexes.

\section{THE MAGNETIC COUPLING IN BINUCLEAR SYSTEMS: BASIC CONCEPTS}

In the valence bond, VB, language (in its formulation based on orthogonal atomic orbitals, OAOs, see (Refs. 15 and 16)), the building blocks of the singlet and triplet wavefunctions for a simple symmetric binuclear complex are (disregarding the core part) as follows:

$$
\begin{aligned}
& \Psi_{N}^{S}=\frac{\|a \bar{b}\|+\|b \bar{a}\|}{\sqrt{2}}, \quad \Psi_{N}^{T}=\frac{\|a \bar{b}\|-\|b \bar{a}\|}{\sqrt{2}}, \\
& \Psi_{I}^{S}=\frac{\|a \bar{a}\|+\|b \bar{b}\|}{\sqrt{2}},
\end{aligned}
$$

where $a$ and $b$ are the two magnetic OAOs, centered on site $\mathrm{A}$ and $\mathrm{B}$, respectively. The first two functions are the singlet $(S)$ and triplet $(T)$ combinations of the two possible electronic structures where $a$ and $b$ are both singly occupied with one $\alpha$ and one $\beta$ electrons (each atom bears one magnetic electron, neutral distributions, $N$ ) and the last function is the plus combination of the two structures where $a$ and $b$ are either doubly occupied, or empty (ionic distributions, I). The localized $a$ and $b$ OAOs can be obtained by the in-phase and out-of-phase combination of the delocalized $g$ and $u$ CASSCF MOs.

The analysis of the factors governing the amplitude of the magnetic coupling constant is often performed by introducing interpretative models based on effective parameters (for a short review see for instance (Refs. 5-7)). The simplest model, based on the Anderson mechanism, ${ }^{17,18}$ introduces three parameters: the direct exchange between the two $a$ and $b$ (local) magnetic orbitals, $K_{a b}$, the hopping integral between the two magnetic centers, $t_{a b}$, and the relative energy of the ionic forms with respect to the neutral ones, $U$. These parameters define $J$ through the equation (based on a pertur- bation approach):

$$
J=2 K_{a b}-\frac{4 t_{a b}^{2}}{U}=J_{\mathrm{F}}+J_{\mathrm{AF}},
$$

where $J_{\mathrm{F}}$ is the ferromagnetic part of $J$ (direct exchange, $2 K_{a b}$ ) and $J_{\mathrm{AF}}$ is the antiferromagnetic part (delocalization effects or kinetic exchange, $-4 t_{a b}{ }^{2} / U$ ). The $K_{a b}, t_{a b}$, and $U$ parameters are easily accessible for a CASCI wavefunction. ${ }^{6}$

The $\Psi_{N}^{S}, \Psi_{N}^{T}$, and $\Psi_{I}^{S}$ functions are used to build the simplest approximations to the singlet $\left(\Psi^{S}\right)$ and triplet $\left(\Psi^{T}\right)$ wavefunctions of the binuclear complex:

$$
\begin{gathered}
\Psi^{S}=C_{N}^{S} \Psi_{N}^{S}+C_{I}^{S} \Psi_{I}^{S}, \quad\left|C_{N}^{S}\right| \gg\left|C_{I}^{S}\right|, \\
\Psi^{T}=\Psi_{N}^{T} .
\end{gathered}
$$

For the singlet wavefunction, (Eq. (3)), the weight of the neutral structure $\left(\left|C_{N}^{S}\right|^{2}\right)$ is much larger than the weight of the ionic one $\left(\left|C_{I}^{S}\right|^{2}\right)$. Here, it is worth noticing that the diagonalization of the Hamiltonian in the simple basis of the three VB structures gives a second singlet state (an excited state) sharing with the magnetic singlet the spatial symmetry,

$$
\Psi_{\mathrm{exc}}^{S}=\lambda \Psi_{N}^{S}+\mu \Psi_{I}^{S},
$$

where $\lambda$ and $\mu$ are related to the coefficients of Eq. (3) $\left(\lambda=C_{I}^{S}\right.$ and $\mu=-C_{N}^{S}$ ). Therefore, in this state, the weight of the ionic structure is largely predominant. This state is referred to in the following as the "second singlet" or the "ionic singlet."

In a MRPT scheme, the coefficients $C_{N}^{S}$ and $C_{I}^{S}$ are defined at the CASCI level and they are not modified by the perturbation approach (up to the fourth order). A method with this characteristic is called "contracted."19 However, in the DDCI wavefunction, the $C_{I}^{S} / C_{N}^{S}$ ratio is largely revised due to the interaction of the ionic forms with the single and double excitations included in the CI matrix. This interaction stabilizes the ionic form, and reduces the $U$ value. Typical values for $U$ at the CASCI level are 20-30 eV, while effective DDCI values are in the range of 5-8 eV. Since the triplet does not contain ionic forms, the stabilization of the ionic forms has a differential effect on the singlet state, in such a way that it gives an important contribution to $J_{\mathrm{AF}}$. This mechanism, however, does not hold in ferromagnetic systems. In these systems, the ionic forms have a negligible weight in the singlet state wavefunction even at the DDCI level (Refs. 7 and 10d), although the excitations contained on the DDCI wavefunctions have a significant impact on the $J$ value. Therefore, the physical factors governing the coupling in the ferromagnetic systems are not intimately connected to the relative energy of the ionic forms, but rather to different mechanisms, such as, for instance, the spin polarization through the bridging ligands. For these reasons, this work deals only with AF compounds, the study of ferromagnetic ones being currently under exploration. Focusing on the AF systems, it is clear that the contracted nature of the MRPT2 approach prevents the revision of the $C_{I}^{S} / C_{N}^{S}$ ratio, and this is a serious problem in the evaluation of $J$.

In order to try to get over this problem, a convenient decontraction procedure for this kind of problems has been proposed in 2006. ${ }^{19}$ It is worth noticing that other decontraction 
approaches, such as, for instance, quasi degenerate perturbation theory, cannot be applied to magnetic systems, given that the ionic singlet state (that involved in the decontraction) is very high in energy and embedded in a set of other states of the system (MLCT, LMCT, etc.). ${ }^{19}$ This fact gives rise to the appearance of quasi degeneracy and therefore of possible divergences in MRPT approaches. The application of the new decontraction procedure has shown a marked increase of the $\left|C_{I}^{S} / C_{N}^{S}\right|$ ratio with respect to the CASCI value (and therefore an increase of the AF character). The agreement with the experimental (or DDCI) $J$ value was improved, but it remained unsatisfactory.

In 2009, a sharp analysis of the effect of the different excitation classes on the variational and perturbation evaluation of $J$ in binuclear $\mathrm{Cu}$ (II) systems has been published. ${ }^{7}$ In this study it has been shown that the contributions of different types of excitations are not additive and they cannot therefore be taken into account in a MRPT2 scheme. LMCT excitations in particular play a key role: “... they contribute at higher order as a correlation induced increase of LMCT components of the wavefunction, i.e., of the mixing between the ligand and the magnetic orbitals." In the conclusion section of Ref. 7, one reads "... the correlation-induced delocalization of the magnetic orbitals ... results in an increase of the absolute value of $J \ldots$ and it becomes evident looking at the natural magnetic orbitals obtained at the DDCI level. This phenomenon is not limited to $1 / 2$ spin systems, but it concerns as well higher spin metallic ions." These considerations have prompted us to thoroughly consider the role of the magnetic orbitals in the calculation of $J$ in MRPT2 approaches.

\section{DESCRIPTION OF THE SYSTEMS AND COMPUTATIONAL DETAILS}

Several binuclear $\mathrm{Cu}$ (II) systems have been considered. The analysis reported in Secs. IV-VI is based on a first set of systems (1-4, Fig. 1), while a second set (systems 5-10, Fig. 1) is considered to further test the strategy proposed in Sec. VII. In all cases, geometries from x-ray crystal data have been used in the calculations. Systems $\mathbf{1}$ and 2 are binuclear fragments of two cuprates, $\mathrm{La}_{2} \mathrm{CuO}_{4}$ (1) and $\mathrm{Sr}_{2} \mathrm{CuO}_{2} \mathrm{Cl}_{2}$ (2), both of them presenting a remarkable antiferromagnetic coupling $\left(-1030,-1096 \mathrm{~cm}^{-1}\right.$ for $\mathrm{La}_{2} \mathrm{CuO}_{4}$ (Refs. 20-23) and $-1008 \mathrm{~cm}^{-1}$ for $\mathrm{Sr}_{2} \mathrm{CuO}_{2} \mathrm{Cl}_{2}$ (Ref. 24)). In both cases, the calculations have been performed on a $\mathrm{Cu}_{2} \mathrm{O}_{7}$ fragment, which coordinates are extracted from the corresponding $\mathrm{x}$-ray crystal structure (Refs. 25 and 26 for $\mathrm{La}_{2} \mathrm{CuO}_{4}$ and $\mathrm{Sr}_{2} \mathrm{CuO}_{2} \mathrm{Cl}_{2}$, respectively). These $\mathrm{Cu}_{2} \mathrm{O}_{7}$ clusters are embedded in a set of point charges and total ion potentials simulating the Pauli repulsion and Madelung potential of the infinite crystal, respectively. The embedding is described in details in previous works (Ref. 27 for $\mathrm{La}_{2} \mathrm{CuO}_{4}$, and Ref. 28 for $\mathrm{Sr}_{2} \mathrm{CuO}_{2} \mathrm{Cl}_{2}$ ). In system 3 (named $\left[\mathrm{Cu}_{2}(\mathrm{OH})_{2}\right]$ ), the $\mathrm{Cu}(\mathrm{II})$ centers are bridged by two hydroxo groups, [(tmen) $\mathrm{Cu}(\mathrm{OH})_{2} \mathrm{Cu}($ tmen $\left.)\right]$, with $\operatorname{tmen}=\mathrm{N}, \mathrm{N}, \mathrm{N}^{\prime}, \mathrm{N}^{\prime}$-tetramethylethylenediamine. ${ }^{29,30}$ The $\mathrm{Cu}(\mathrm{II})$ centers present an antiferromagnetic coupling, with $J=-509 \mathrm{~cm}^{-1}$. System 4 corresponds to an oxalate bridged binuclear $\mathrm{Cu}(\mathrm{II})$ compound [ $\left.\mathrm{LCu}-\left(\mathrm{C}_{2} \mathrm{O}_{4}\right)-\mathrm{CuL}\right]$
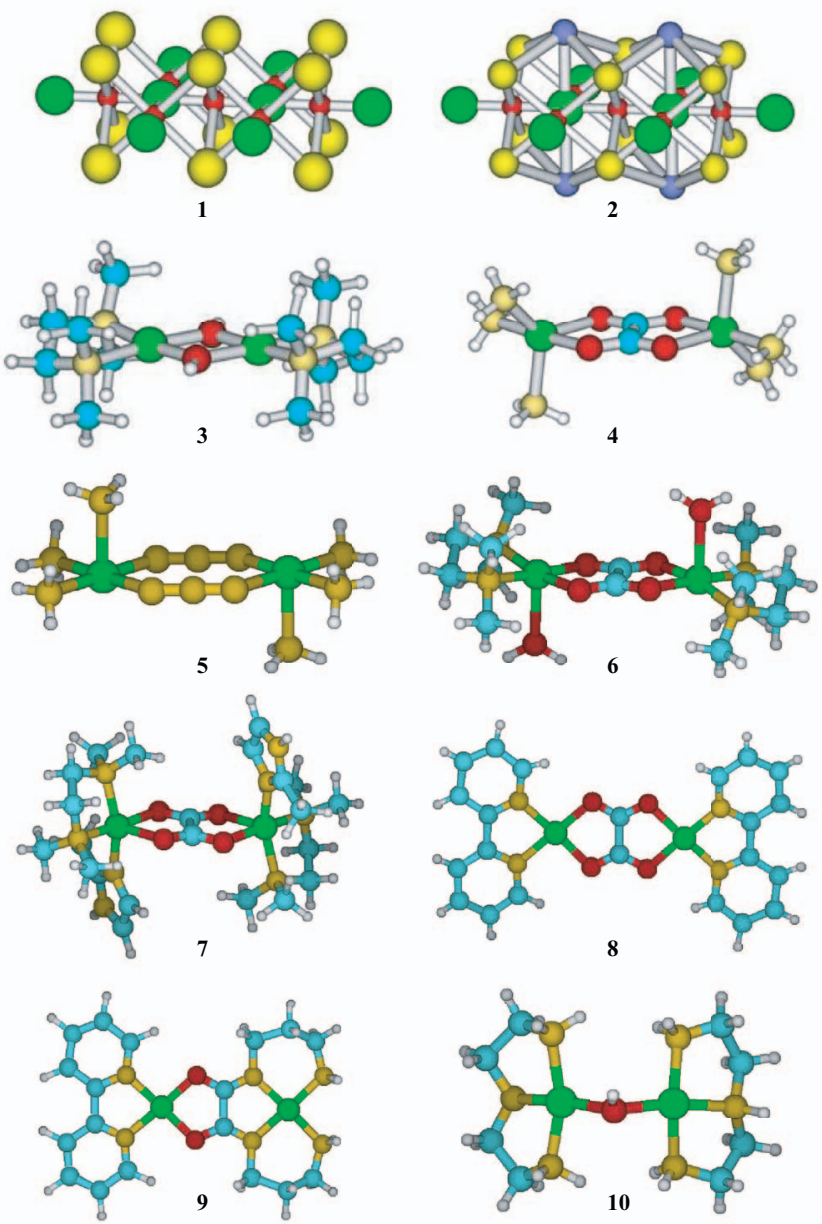

FIG. 1. Structures of the systems under consideration. Color coding for the atoms: copper, green; carbon, light blue; oxygen, red; nitrogen, yellow; chlorine, dark blue; hydrogen, white.

with $\mathrm{L}=1,1,4,7,7$-pentaethyldiethylene-triamine, also antiferromagnetic, ${ }^{31}$ with $J=-75 \mathrm{~cm}^{-1}$. In this structure, the external ligands have been modeled with $\mathrm{NH}_{3}$ groups, with the coordinated $\mathrm{N}$ at the experimental position. The resulting model is named $\left[\mathrm{Cu}_{2}\left(\mathrm{C}_{2} \mathrm{O}_{4}\right)\right]$, hereafter. In system 5, with formula $\left[\mathrm{LCu}-(\mathrm{NNN})_{2}-\mathrm{CuL}\right]$, where $\mathrm{L}=\mathrm{N}, \mathrm{N}^{\prime}, \mathrm{N}^{\prime \prime}-$ trimethyl-1,4,7-triazacyclononane, the bridging ligands correspond to two end-to-end azido groups and it is indicated with $\left[\mathrm{Cu}-\left(\mathrm{N}_{3}\right)_{2}-\mathrm{Cu}\right]$ in the following. This coordination produces a large stabilization of the singlet state $(J<-800$ $\left.\mathrm{cm}^{-1}\right){ }^{32}$ Systems 6-8 are also oxalate bridged complexes, with formula [tmen $\left.\left(\mathrm{H}_{2} \mathrm{O}\right) \mathrm{Cu}\left(\mathrm{C}_{2} \mathrm{O}_{4}\right) \mathrm{Cu}\left(\mathrm{H}_{2} \mathrm{O}\right) \operatorname{tmen}\right]\left(\mathrm{ClO}_{4}\right)_{2}$, [tmen $(2-\mathrm{MeIm}) \mathrm{Cu}\left(\mathrm{C}_{2} \mathrm{O}_{4}\right) \mathrm{Cu}(2-\mathrm{MeIm})$ tmen] $\left(\mathrm{PF}_{6}\right)_{2}$, and [(bipy) $\left(\mathrm{NO}_{3}\right)\left(\mathrm{H}_{2} \mathrm{O}\right) \mathrm{Cu}\left(\mathrm{C}_{2} \mathrm{O}_{4}\right) \mathrm{Cu}\left(\mathrm{H}_{2} \mathrm{O}\right)\left(\mathrm{NO}_{3}\right)($ bipy)$)$, respectively, with 2-MeIm=2-methylimidazole and bipy $=2,2^{\prime}$ bipyridine (these three systems are indicated hereafter with $[\mathrm{Cu}-(\mathrm{ox})-\mathrm{Cu}])$. They present AF couplings with $J$ values of $-385.4 \mathrm{~cm}^{-1}$ (Ref. 33), $-13.8 \mathrm{~cm}^{-1}$ (Ref. 33), and $-382 \mathrm{~cm}^{-1}$ (Ref. 34). In system 8, neither the $\mathrm{NO}_{3}$ groups, nor the $\mathrm{H}_{2} \mathrm{O}$ are included in the calculations. In system 9 ([Cu-(oxpn)- $\mathrm{Cu}]$ hereafter), the two $\mathrm{Cu}$ ions are bridged by an oxamido bridge, with formula $[\mathrm{Cu}($ oxpn $) \mathrm{Cu}($ bipy $)]\left(\mathrm{ClO}_{4}\right)_{2}$, oxpn $=N, N^{\prime}$-bis(3-aminopropyl)oxamido, where $J$ was found to be equal to $-439.7 \mathrm{~cm}^{-1}$ (Ref. 35). Finally, system 10 
contains only one hydroxo group bridging two $\mathrm{LCu}$ moieties, with $\mathrm{L}=$ diethylenetriamine ([Cu- $(\mathrm{OH})-\mathrm{Cu}])$. This system presents a large singlet-triplet gap with $J=-374 \mathrm{~cm}^{-1}$ (Ref. 36).

In structures 1-3 and 5, the core electrons of $\mathrm{Cu}$ atoms (up to $3 \mathrm{~s}$ ) have been replaced with effective core potentials, and the $(9 s 6 p 6 d) /[3 s 3 p 4 d]$ basis set has been used for the valence electrons of $\mathrm{Cu}$. For system $4, \mathrm{Cu}$ atoms have been represented by means of atomic natural orbital (ANO)-type basis functions with contractions [5s $4 \mathrm{p} 3 \mathrm{~d} 1 \mathrm{f}]$, on the basis of our previous experience with this system. ${ }^{37}$ In the rest of systems, contractions [5s4p3d] have been employed for $\mathrm{Cu}$ atoms.

Regarding the ligands, for systems $\mathbf{1}$ and $\mathbf{2}, \mathrm{O}$ atoms basis set is of DZP quality with contractions (10s5p1d)/[3s2p1d] as in previous works. ${ }^{27}$ For the remaining systems, ANO-type basis functions of different quality are used for the ligands, depending on the specific position. Contractions [3s2p1d] are employed for $\mathrm{C}, \mathrm{N}$, and $\mathrm{O}$ in bridging positions, and [4s3p1d] for bridging $\mathrm{Cl}$. For the external ligands, contractions [3s2p] are used for atoms directly bonded to the metal centers and [2s1p] for the remaining positions. $\mathrm{H}$ atoms are represented by means of ANO-type functions with a contraction [2s1p] when they are placed on the bridging ligands, and $2 \mathrm{~s}$ or $1 \mathrm{~s}$ for the rest. ${ }^{38-40}$ All calculations on the same system have been done with the same basis set to make the comparison of different methods meaningful.

DDCI calculations have been performed by means of CASDI code ${ }^{41,42}$ while the NEVPT2 method ${ }^{11-14}$ on the minimal $(2,2)$ CAS has been employed to evaluate the perturbation corrections to the energy, in both the partially contracted (PC) and strongly contracted (SC) versions. For the sake of comparison, also CASPT2 calculations have been carried out by using the IPEA $=0$ option implemented in the MOLCAS 7.6 package. ${ }^{43}$ This is not the standard $\hat{\mathcal{H}}_{0}$ for the last versions of CASPT2, but some studies have shown that it is preferable for the calculation of $J$ (see Ref. 4b).

Regarding the molecular orbitals (MOs), different sets have been employed. The first set is obtained from a $\operatorname{CASSCF}(2,2)$ calculation on the magnetic singlet state. To check the impact of the dynamic correlation, the natural orbitals of the average DDCI density matrix of the singlet and triplet states (both computed on top of the singlet CASSCF $(2,2)$ MOs) have been also considered. ${ }^{27,44,45}$ The so-resulting MOs are called DDCI natural orbitals. Finally, a state-average CASSCF procedure (SA-CASSCF) is employed to obtain the orbital to be used in MRPT2 calculations. The procedure mixes the two lowest singlet states, with large projections on the neutral (first state) and ionic (second state) VB structures, at $\operatorname{CASSCF}(2,2)$ level. The weight of each state in the state-average procedure is a key question for the perturbation evaluation of $J$. This point is addressed in detail in Sec. V.

\section{A CLOSE INSPECTION OF THE DDCI NATURAL MOS}

In the calculation of $J$ within a MRPT2 approach, the orbitals are in general obtained with a CASSCF calculation on either the triplet, or the singlet. As an example, in Table I,
TABLE I. Magnetic coupling constant $J\left(\mathrm{~cm}^{-1}\right)$ computed from the DDCI and MRPT2 methods using the CASSCF $(2,2)$ singlet MOs and the DDCI natural MOs

\begin{tabular}{lcccc}
\hline \hline & $\begin{array}{c}\mathrm{La}_{2} \mathrm{CuO}_{4} \\
(\mathbf{1})\end{array}$ & $\begin{array}{c}\mathrm{Sr}_{2} \mathrm{CuO}_{2} \mathrm{Cl}_{2} \\
\text { (2) }\end{array}$ & $\begin{array}{c}\left.\mathrm{Cu}_{2}(\mathrm{OH})_{2}\right] \\
(\mathbf{3})\end{array}$ & $\begin{array}{c}{\left[\mathrm{Cu}_{2}\left(\mathrm{C}_{2} \mathrm{O}_{4}\right)\right]} \\
(\mathbf{4})\end{array}$ \\
\hline CASCI & -387 & -237 & -72 & -10 \\
SC-NEVPT2 & -587 & -363 & -129 & -18 \\
PC-NEVPT2 & -605 & -380 & -138 & -20 \\
CASPT2 & -711 & -480 & -200 & -35 \\
DDCI & -1149 & -1002 & -523 & -80 \\
& & DDCI natural MOs & \\
CASCI & -468 & -292 & 17 & -6 \\
SC-NEVPT2 & -1006 & -896 & -471 & -90 \\
PC-NEVPT2 & -1029 & -924 & -493 & -97 \\
CASPT2 & -1162 & -1311 & -1011 & -247 \\
DDCI & -1145 & -1010 & -473 & -119 \\
Exp. & $-1030,-1096$ & -1008 & -509 & -75 \\
\hline \hline
\end{tabular}

the results obtained with NEVPT2 (SC and PC) and CASPT2 starting from the singlet CASSCF orbitals are reported for a representative subset of the systems considered in this work. These results confirm the quality of the DDCI approach and that too low absolute values of $J$ (with respect to the experimental and DDCI values) are computed at MRPT2 level when a minimal CAS is employed. Similar results are obtained using the triplet CASSCF orbitals or state-specific orbitals. ${ }^{7}$

To investigate the importance of the starting orbitals, the same calculations have been performed also using the DDCI natural orbitals (see Table I). Passing from the singlet MOs to the DDCI natural MOs, one notes that at the DDCI level there is an almost negligible effect on $J$ for $\mathbf{1}$ and $\mathbf{2}$, the variation is small for $\mathbf{3}$, and slightly more remarkable for $\mathbf{4}$. On the contrary, a large variation of the $J$ values is found at PT2 level for all four systems. In the last case, the agreement with DDCI and experiment is improved when using the DDCI natural MOs, in particular for the NEVPT2 values. It is worth stressing again that the use of the singlet MOs or of statespecific MOs has only a minor effect on the $J$ values computed at NEVPT2 level.

The use of the DDCI natural MOs in a MRPT2 calculation is manifestly not a computational strategy, given that an expensive DDCI step is required to compute the orbitals for a much faster MRPT2 step and that the DDCI step already provides good values of $J$. Nevertheless, this result points out the importance of the starting MOs and allows us to suppose that with an appropriate choice of the MOs, the MRPT2 approach on the minimal CAS can give reasonable $J$ values. This consideration is one of the key points of the present work, given that the orbitals have not been considered in the past as one of the pivotal aspects in this field.

In order to understand the origin of this behavior and possibly devise an effective computational strategy, one has to address two key questions:

1. What physical effect is correctly taken into account in the DDCI natural MOs and largely underestimated in the CASSCF MOs? 
2. Is it possible to compute a set of MOs considering this effect at a lower (computationally less expensive) level than DDCI?

The answer to the second question is possible only after answering the first question and it is the subject of Sec. V. The first question is primarily conceptual and to answer it one has to reconsider the nature of the minimal active space CASSCF wavefunctions (both the triplet and the singlet ones). They are purely neutral (triplet state) or essentially neutral (singlet state) and the CASSCF procedure leads to MOs optimized for these wavefunctions. The final orbitals are a good solution for the neutral VB structures but they very badly describe the ionic VB forms. This bias against the ionic forms at the CASSCF level can be obviously remedied in post CASSCF methods, such as DDCI or MRPT, but the analysis of the wavefunctions obtained with these methods is undoubtedly complex. However, one can show that a more balanced treatment of ionic and neutral VB forms can be obtained by a relaxation of the CASSCF MOs. Orbital modifications are introduced by single excitations on the top of the CASSCF wavefunction, but these excitations do not directly interact with the CASSCF wavefunction as stated by the generalized Brillouin theorem $^{46}$ (the orbitals are optimized for this wavefunction and orbital modification is not required if the wavefunction is not changed). For this reason, orbital relaxation is not considered in MRPT2 or in MRPT3, since the numerators of the perturbation series contain the direct interaction of the singly excited perturbation functions with the CASSCF wavefunction. It is possible to show that the orbital relaxation is taken into account only at higher order, where single excitations appear in terms where they interact with double excitations, which in turn interact with the CASSCF wavefunction. This effect can be identified as an orbital relaxation induced by the electron correlation. The inability of MRPT applied to second and third order for the energy (first order for the wavefunction) to consider such an effect has been recently identified in a different context, the calculation of the $\pi \rightarrow \pi^{*}$ excited states of ethylene. ${ }^{47}$ It is worth noticing that, unlike MRPT, variational methods (as DDCI) can describe the orbital relaxation induced by the electron correlation.

As the nature of the orbital relaxation is concerned, one can reasonably expect that a balanced description of ionic and neutral forms requires more "diffuse" (delocalized) orbitals than the CASSCF ones, and this is actually a property of the natural DDCI MOs, as commented in details in Ref. 7 and at the end of Sec. II. In Sec. V, we address the development of an effective and computational not expensive definition of a set of MOs to be used in MRPT2 which fulfills this requirement.

\section{AN EFFECTIVE STRATEGY TO COMPUTE THE ORBITALS}

Our proposal to obtain the orbitals to be used in the MRPT2 calculation of $J$ is to compute them by a state-average CASSCF (SA-CASSCF) procedure involving the magnetic singlet and the second singlet of the same symmetry. This singlet is essentially ionic (with a small neutral component, see Sec. II and Eq. (5)) and therefore, the state-average energy minimization produces MOs well suited for both the neutral and ionic VB structures. The key point to tackle is the correct value for the weights in the state-average procedure. To guarantee that the two forms are treated at the same level, one can suppose that the weights must be $0.5 / 0.5$. Actually, the CASSCF wavefunction misses different physical effects. Among them, one can identify effects whose absence makes the MOs of a pure ionic wavefunction too diffuse (dynamic polarization of the ionic forms, ${ }^{47}$ dynamic correlation of the two magnetic electrons) and for these reasons, the magnetic orbitals of a SA-CASSCF calculation with equal weights are too diffuse. This defect can be remedied if the weight of the second singlet is lower than that of the magnetic singlet, but the correct weight cannot be defined a priori. To verify the reasonableness of these considerations, we have computed the overlap of the SA-CASSCF magnetic MOs with the DDCI natural magnetic MOs (the "best" MOs in hand, which give the improved values of $J$ in MRPT2, see Sec. IV and Table I) for different weights of the magnetic singlet in the SA-CASSCF procedure (hereafter indicated with $W$ ). The results are reported in Fig. 2 for the four systems considered in this section (1-4).

In all cases, the considerations reported above are confirmed:

- the CASSCF magnetic MOs of the magnetic singlet ( $W=100 \%$ in Fig. 2) are rather different from the best MOs;

- the agreement between the two sets of MOs is regularly improved if the second (ionic) singlet is included in the optimization procedure with increasing weight;

- the overlap reach a maximum for $W \simeq 0.7$ (it is close to the maximum for $0.66<W<0.74$ ) and then the agreement decreases, showing that the SA-CASSCF procedure with equal weight overestimates the delocalization of the active MOs.

Besides this confirmation, a surprising result can be noted from Fig. 2: the maximum overlaps are very close to 1 . This allows to conclude that, for these systems, with a simple SACASSCF (2/2) calculation one can closely reproduce the natural magnetic orbitals of two expensive DDCI calculations. This further confirms that the main modification, moving from the magnetic singlet CASSCF MOs to the DDCI natural MOs, is an increase of the spatial extent of the magnetic MOs, required to enable a balanced description of the neutral and ionic VB forms. The SA-CASSCF orbitals computed for $W=0.7$ (a value for which the overlap with the DDCI natural MOs is close to one) are indicated hereafter with SA-7/10 MOs. The three key sets of orbitals (DDCI natural, singlet CASSCF, and SA-7/10) are depicted in Fig. 3 for system 1 $\left(\mathrm{La}_{2} \mathrm{CuO}_{4}\right)$.

\section{THE EFFECT OF THE STATE AVERAGE PROCEDURE ON THE $J$ VALUE AT MRPT2 LEVEL}

The dependence of the MRPT2 $J$ value on the weight, $W$, of the magnetic singlet used to define the orbitals is reported in Fig. 4 for the complexes 1-4. From this figure, it is apparent that the MRPT2 $J$ value strongly depends on $W$ : when 

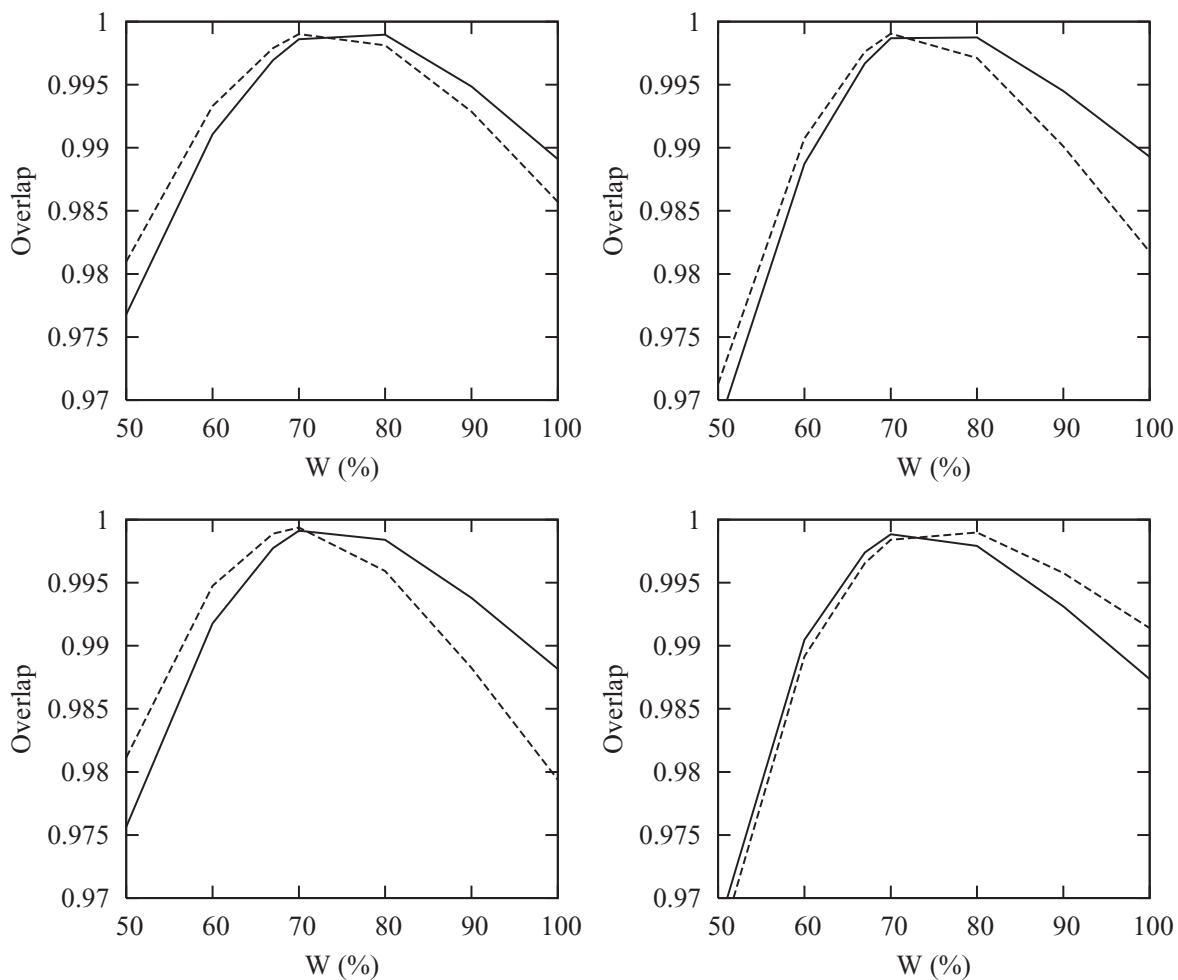

FIG. 2. Overlap of the two SA-CASSCF magnetic MOs (full and dashed lines) with the DDCI natural magnetic MOs as a function of the weight of the magnetic singlet in the SA-CASSCF procedure $(W)$ for system $\mathbf{1}\left(\mathrm{La}_{2} \mathrm{CuO}_{4}\right.$ (top left)), $\mathbf{2}\left(\mathrm{Sr}_{2} \mathrm{CuO}_{2} \mathrm{Cl}_{2}\right.$ (top right)), $3\left(\left[\mathrm{Cu}_{2}(\mathrm{OH})_{2}\right]\left(\right.\right.$ bottom left)), and $\mathbf{4}\left(\left[\mathrm{Cu}_{2}\left(\mathrm{C}_{2} \mathrm{O}_{4}\right)\right]\right.$ (bottom right)).

the weight of the ionic singlet is increased ( $W$ decreases), the absolute value of $J$ increases, raising the antiferromagnetic character of the magnetic coupling. This behavior is found for both the NEVPT2 (SC-NEVPT2 and PC-NEVPT2 giving
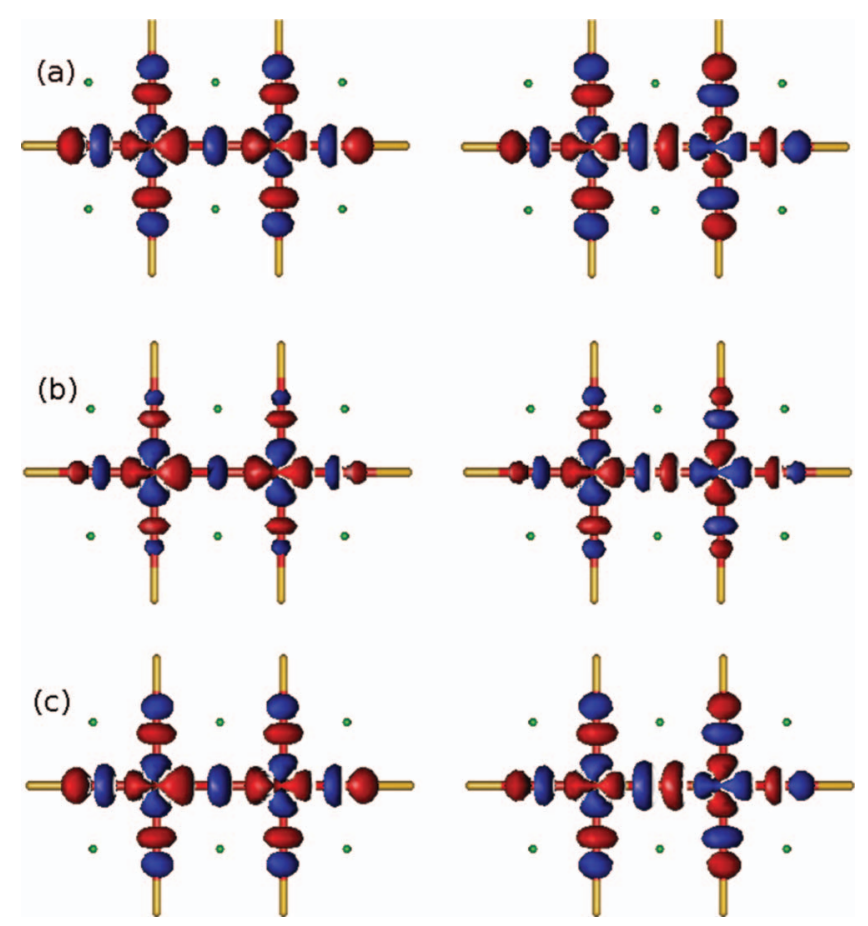

FIG. 3. Symmetry-adapted $g$ and $u$ magnetic orbitals for system $\mathbf{1}$ $\left(\mathrm{La}_{2} \mathrm{CuO}_{4}\right)$ : The DDCI natural MOs (a, top), the singlet CASSCF MOs (b, middle), and the SA-7/10 MOs (c, bottom). almost the same value) and the CASPT2 approaches (with the only exception of the CASPT2 values for system $\mathbf{3}$ at low values of $W$ ).

The comparison of this trend with that observed at the CASCI level, (not reported here, the $J$ values are almost unchanged for $W$ in the range $60 \%-100 \%$ ), reveals that the effects introduced by the dynamical electron correlation strongly depend on $W$. In fact, the stabilization of the ionic forms is a crucial requirement for a quantitative evaluation of $J$, since these forms participate in the dominant mechanisms giving the largest contributions to $J$ (Refs. (5-7)). When we enter to the perturbation treatment with the singlet or triplet CASSCF orbitals, the ionic forms are too high in energy ( $U$ around $24 \mathrm{eV}$ for the systems considered), and the contributions to $J$ coming from the interaction of the ionic forms with the $2 \mathrm{~h} 1 \mathrm{p}$ excitations (the leading ones) are energetically penalized (denominators in the perturbation expression too large). Then, the resulting MRPT2 $J$ value is too small compared with the DDCI or experimental ones. The larger the stabilization of these forms introduced by smaller $W$, the lower the penalty, the larger the impact on the $J$ value, as shown in Fig. 4.

\section{EVALUATION OF J FROM MRPT2 CALCULATION USING THE SA-7/10 MOS}

On the basis of these considerations, we propose a computational strategy for the calculation of the magnetic coupling constant in the frame of MRPT2 methods: 

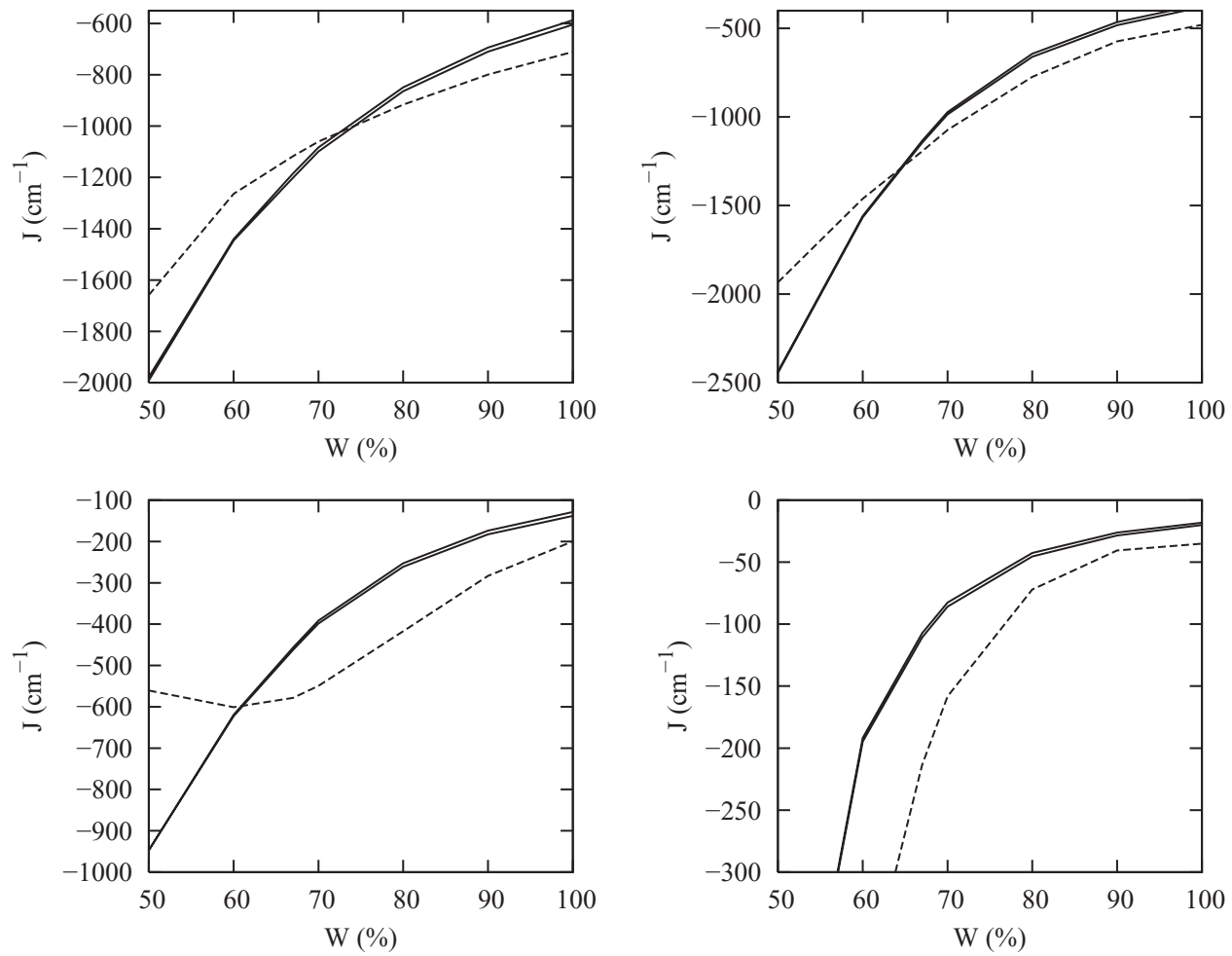

FIG. 4. Dependence of the MRPT2 $J$ value on the weight $W$ of the magnetic singlet in the SA procedure for systems $1\left(\mathrm{La}_{2} \mathrm{CuO}_{4}(\mathrm{top}\right.$ left $\left.)\right), 2\left(\mathrm{Sr}_{2} \mathrm{CuO}_{2} \mathrm{Cl}_{2}\right.$ (top right)), $\mathbf{3}\left(\left[\mathrm{Cu}_{2}(\mathrm{OH})_{2}\right]\right.$ (bottom left)), and $\mathbf{4}\left(\left[\mathrm{Cu}_{2}\left(\mathrm{C}_{2} \mathrm{O}_{4}\right)\right]\right.$ (bottom right)). Full lines, SC- and PC-NEVPT2 values; dashed line, CASPT2 values.

- the MOs are computed at SA-CASSCF level for the two singlets with weights 0.7 and 0.3 on the magnetic and ionic singlets, respectively;

- the $J$ value is obtained from the difference of the MRPT2 energies of the magnetic singlet and triplet states, where the zero order wavefunctions are of CASCI type computed from the SA-7/10 MOs.

The results of such a strategy for the systems considered in Secs. IV-VI are reported in Table II. To give an estimation of the dependence of these values on small variations of $W$, we also report the results obtained with $W=0.66$ and $W=0.74$ (values within parenthesis), given that in this interval the overlap of the SA-CASSCF orbitals with the DDCI natural ones is very close to the maximum value, as discussed in Sec. $\mathrm{V}$ and shown in Fig. 2.

The $J$ values are now in a reasonable agreement with the experimental estimations for both NEVPT2 and CASPT2. The largest deviations are found for system $\mathbf{3}$ at NEVPT2 level and for system 4 at CASPT2 level. The improvement with respect to the results obtained starting from the CASSCF MOs of the magnetic singlet is large (compare the values of Table II with those of Table I). Moreover, they closely resemble those obtained starting from the high level DDCI natural orbitals.

To further confirm the quality of the computational strategy here proposed, it has been applied on six more binuclear $\mathrm{Cu}$ complexes (systems 5-10). Systems 9 and $\mathbf{1 0}$ are not

TABLE II. Magnetic coupling constant $J\left(\mathrm{~cm}^{-1}\right)$ computed at the DDCI and MRPT2 level starting from the CAS $(2,2)$ space and using the SA-7/10 MOs. Values computed with $W=0.66$ and $W=0.74$ within parenthesis.

\begin{tabular}{lcccc}
\hline \hline & $\begin{array}{c}\mathrm{La}_{2} \mathrm{CuO}_{4} \\
(\mathbf{1})\end{array}$ & $\begin{array}{c}\mathrm{Sr}_{2} \mathrm{CuO}_{2} \mathrm{Cl}_{2} \\
\text { (2) }\end{array}$ & $\begin{array}{c}{\left[\mathrm{Cu}_{2}(\mathrm{OH})_{2}\right]} \\
(\mathbf{3})\end{array}$ & $\begin{array}{c}{\left[\mathrm{Cu}_{2}\left(\mathrm{C}_{2} \mathrm{O}_{4}\right)\right]} \\
(\mathbf{4})\end{array}$ \\
\hline $\begin{array}{l}\text { SA-7/10 MOs } \\
\text { DDCI }\end{array}$ & -1133 & -1022 & -495 & -118 \\
SC-NEVPT2 & -1067 & -971 & -392 & -83 \\
& $(-967,-1187)$ & $(-816,-1167)$ & $(-322,-466)$ & -398 \\
PC-NEVPT2 & -1084 & -984 & $(-330,-471)$ & $(-65,-117)$ \\
& $(-982,-1204)$ & $(-831,-1177)$ & -549 \\
CASPT2 & -1041 & -1065 & $(-502,-583)$ \\
Exp. & $(-985,-1104)$ & $(-938,-1201)$ & -509 \\
\hline \hline
\end{tabular}


TABLE III. Magnetic coupling constant $J\left(\mathrm{~cm}^{-1}\right)$ computed at the DDCI and MRPT2 level starting from the CAS $(2,2)$ space and using the SA-7/10 MOs. Values computed with $W=0.66$ and $W=0.74$ within parenthesis.

\begin{tabular}{|c|c|c|c|c|c|c|}
\hline Systems & $\begin{array}{c}{\left[\mathrm{Cu}-\left(\mathrm{N}_{3}\right)_{2}-\mathrm{Cu}\right]} \\
\mathbf{5}\end{array}$ & $\begin{array}{c}{[\mathrm{Cu}-(\mathrm{ox})-\mathrm{Cu}]} \\
\mathbf{6}\end{array}$ & $\begin{array}{c}{[\mathrm{Cu}-(\mathrm{ox})-\mathrm{Cu}]} \\
7\end{array}$ & $\begin{array}{c}{[\mathrm{Cu}-(\mathrm{ox})-\mathrm{Cu}]} \\
\mathbf{8}\end{array}$ & $\begin{array}{c}{[\mathrm{Cu}-(\text { oxpn })-\mathrm{Cu}]} \\
9\end{array}$ & $\begin{array}{c}{[\mathrm{Cu}-\mathrm{OH}-\mathrm{Cu}]} \\
\mathbf{1 0}\end{array}$ \\
\hline & \multicolumn{6}{|c|}{ SA-7/10 MOs } \\
\hline DDCI & -1136 & -290 & -15 & -347 & -376 & -231 \\
\hline SC-NEVPT2 & $\begin{array}{c}-1095 \\
(-785,-1540)\end{array}$ & $\begin{array}{c}-194 \\
(-160,-237)\end{array}$ & $\begin{array}{c}-13 \\
(-9,-19)\end{array}$ & $\begin{array}{c}-217 \\
(-176,-271)\end{array}$ & $\begin{array}{c}-260 \\
(-210,-323)\end{array}$ & $\begin{array}{c}-270 \\
(-226,-326)\end{array}$ \\
\hline PC-NEVPT2 & $\begin{array}{c}-1114 \\
(-804,-1559)\end{array}$ & $\begin{array}{c}-201 \\
(-167,-244)\end{array}$ & $\begin{array}{c}-13 \\
(-9,-20)\end{array}$ & $\begin{array}{c}-226 \\
(-185,-280)\end{array}$ & $\begin{array}{c}-274 \\
(-223,-338)\end{array}$ & $\begin{array}{c}-279 \\
(-235,-334)\end{array}$ \\
\hline \multirow[t]{2}{*}{ CASPT2 } & $\begin{array}{c}-1954 \\
(-1382,-2635)\end{array}$ & $\begin{array}{c}-244 \\
(-211,-285)\end{array}$ & $\ldots^{a}$ & $\begin{array}{c}-329 \\
(-270,-402)\end{array}$ & $\begin{array}{c}-364 \\
(-305,-431)\end{array}$ & $\begin{array}{c}-344 \\
(-304,-392)\end{array}$ \\
\hline & \multicolumn{6}{|c|}{ Singlet MOs } \\
\hline DDCI & -1116 & -246 & -12 & -280 & -280 & -237 \\
\hline SC-NEVPT2 & -184 & -63 & -2 & -65 & -70 & -95 \\
\hline PC-NEVPT2 & -198 & -68 & -2 & -71 & -77 & -104 \\
\hline CASPT2 & -336 & -99 & $\ldots^{\mathrm{a}}$ & -106 & -117 & -142 \\
\hline Exper. & $<-800$ & -385 & -14 & -382 & -440 & -374 \\
\hline
\end{tabular}

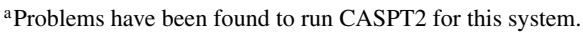

symmetric and therefore, two ionic singlets can be computed with the minimal active space (corresponding to the $g$ and $u$ ionic forms in symmetric systems). In this case, the SA7/10 MOs are computed in a SA-CASSCF calculations with a weight 14 on the magnetic singlet and 3:3 on the two other singlets. The $J$ values computed at MRPT2 level starting from the SA-7/10 MOs are reported in Table III, together with the values computed starting from the $\operatorname{CASSCF}(2,2)$ orbitals of the singlet. These results validate what observed for systems 1-4. Passing from the $\operatorname{CASSCF}(2,2)$ singlet MOs to the SA-7/10 MOs, there is a marked improvement of the quality of the $J$ values, which in the last case are in a better agreement with both the experimental and the DDCI values.

\section{CONCLUSIONS}

In this contribution, we have presented some evidences of the fact that the failure of MRPT2 evaluations of $J$ based on minimal active spaces is just a particular manifestation of a general problem, that is, the "orbital relaxation induced by the electron correlation." This problem appears in different situations, such as, for instance, the ionic excited states of $\pi$ conjugated molecules. In CI methods, the orbital relaxation is introduced naturally as a result of the interaction of the reference wavefunction with the excited determinants (provided that an uncontracted scheme is employed, as DDCI). In the case of MRPT methods, the orbital relaxation is not considered at order lower than four, while most of the routine applications rest on PT2 level. To overcome this difficulty, extended CAS are often employed containing the $d^{\prime}$ shell as well as bridge ligand orbitals, which allow for the relaxation of the ionic forms and introduce the MLCT and LMCT forms, respectively.
In the present work, we have shown that this relaxation effect can be alternatively included by using a well-adapted MOs set, in particular, a set obtained from the average of the two lowest singlet states, which are essentially neutral and ionic in nature. A 7:3 mixture gives magnetic orbitals showing a large overlap ( $>0.995)$ with the DDCI natural MOs. This can be considered as a guarantee that the main relaxation effects are taken into account in the so-obtained MOs set. NEVPT2 calculations using these MOs and a minimal active space spanned by the SA-7/10 magnetic orbitals give $J$ amplitudes in acceptable agreement with the DDCI and experimental ones, while the same calculations with state specific MOs provide very poor estimates of $J$.

One can therefore conclude that the strategy here proposed, based on a detailed analysis of the factor governing the nature of the magnetic MOs in different types of wavefunctions, is grounded and allows for the computation of the magnitude of the magnetic coupling constant with MRPT2 methods with a reasonable accuracy in antiferromagnetic systems. For medium size molecular systems, for which DDCI calculations are affordable, DDCI remains the natural choice. The here suggested approach presents itself as an interesting candidate for large systems, for which DDCI becomes prohibitively costly and usual MRPT2 evaluations are limited by the "bottleneck" of the CASSCF calculations with very large active spaces.

Few remarks are worthy at this point. All systems here studied are antiferromagnetic, where the relaxation of the ionic forms is a key process for the coupling between the magnetic centers. For ferromagnetic systems, the stabilization of the ionic forms has a negligible impact on the singlet wavefunction and therefore, the strategy here proposed does not improve significantly the quality of the MRPT2 approach based on a minimal active space. Different strategies, which 
take into account the main features of the ferromagnetic systems, are currently under exploration, with the aim to establish a computational strategy similar to that here proposed for AF systems. Also, it would be useful to extend this procedure to polynuclear systems with more than two magnetic metal centers and/or more than one unpaired electron per magnetic center. This extension is not trivial and works are in progress to evaluate how to face this problem.

\section{ACKNOWLEDGMENTS}

This work has been financed by the the University of Ferrara through its local funding and by the Spanish Ministry of Science and Innovation (Project No. CTQ2009-07767).

This paper is dedicated to Ria Broer on the occasion of her 60th birthday.

${ }^{1}$ R. Broer and W. J. A. Maaskant, Chem. Phys. 102, 103 (1986).

${ }^{2}$ J. Miralles, J. P. Daudey, and R. Caballol, Chem. Phys. Lett. 198, 555 (1992).

${ }^{3}$ J. Miralles, O. Castell, R. Caballol, and J.-P. Malrieu, Chem. Phys. 172, 33 (1993).

${ }^{4}$ Some examples of applications of DDCI method to the evaluation of $J$ : (a) J. B. Rota, L. Norel, C. Train, N. Ben Amor, D. Maynau, and V. Robert, J. Am. Chem. Soc. 130, 10380 (2008); (b) N. Queralt, D. Taratiel, C. de Graaf, R. Caballol, R. Cimiraglia, and C. Angeli, J. Comp. Chem. 29, 994 (2008); (c) C. J. Calzado, Mol. Phys. 35, 1057 (2009); (d) C. J. Calzado, A. Monari, and S. Evangelisti, J. Comp. Chem. 32, 315 (2011).

${ }^{5}$ C. J. Calzado, J. Cabrero, J.-P. Malrieu, and R. Caballol, J. Chem. Phys. 116, 2728 (2002).

${ }^{6}$ C. J. Calzado, J. Cabrero, J.-P. Malrieu, and R. Caballol, J. Chem. Phys. 116, 3985 (2002).

${ }^{7}$ C. J. Calzado, C. Angeli, D. Taratiel, R. Caballol, and J.-P. Malrieu, J. Chem. Phys. 131, 044327 (2009).

${ }^{8}$ C. J. Calzado and D. Maynau, J. Chem. Phys. 135, 194704 (2011).

${ }^{9}$ K. Andersson, P.-Å. Malmqvist, B. O. Roos, A. J. Sadlej, and K. Wolinski, J. Chem. Phys. 94, 5483 (1990).

${ }^{10}$ Some recent examples of the applications of CASPT2 approach to the evaluation of $J$ : (a) Y. Ma, N. A. G. Bandeira, V. Robert, and E. Q. Gao, Chem.-Eur. J 17, 1988 (2011); (b) S. Vancoillie, L. Rulisek, F. Neese, and K. Pierloot, J. Phys. Chem. A 113, 6149 (2009); (c) R. Maurice, A. M. Pradipto, N. Guihery, R. Broer, and C. de Graaf, J. Chem. Theory Comput. 6, 3092 (2010); (d) C. J. Calzado, C. Angeli, C. de Graaf, and R. Caballol, Theor. Chem. Acc. 128, 505 (2011).

${ }^{11}$ C. Angeli, R. Cimiraglia, S. Evangelisti, T. Leininger, and J.-P. Malrieu, J. Chem. Phys. 114, 10252 (2001).

${ }^{12}$ C. Angeli, R. Cimiraglia, and J.-P. Malrieu, Chem. Phys. Lett. 350, 297 (2001).

${ }^{13}$ C. Angeli, R. Cimiraglia, and J.-P. Malrieu, J. Chem. Phys. 117, 9138 (2002).

${ }^{14}$ C. Angeli, M. Pastore, and R. Cimiraglia, Theor. Chem. Acc. 117, 743 (2007).

${ }^{15}$ J.-P. Malrieu, N. Guihéry, C. J. Calzado, and C. Angeli, J. Comput. Chem. 28, 35 (2007).

${ }^{16}$ C. Angeli, R. Cimiraglia, and J.-P. Malrieu, J. Chem. Educ. 85, 150 (2008).

${ }^{17}$ P. W. Anderson, Phys. Rev. 79, 350 (1950).
${ }^{18} \mathrm{P} . \mathrm{W}$. Anderson, Theory of the Magnetic Interaction: Exchange in Insulators and Superconductors, Solid State Physics Vol. 14, edited by F. Turnbull and F. Seitz (Academic, New York, 1963), p. 99.

${ }^{19}$ C. Angeli, C. J. Calzado, R. Cimiraglia, and J.-P. Malrieu, J. Chem. Phys. 124, 234109 (2006).

${ }^{20}$ P. E. Sulewski, P. A. Fleury, K. B. Lyons, S. W. Cheong, and Z. Fisk, Phys. Rev. B 41, 225 (1990).

${ }^{21}$ R. P. Singh, P. A. Fleury, K. B. Lyons, and P. C. Sulewski, Phys. Rev. Lett. 62, 2736 (1989).

${ }^{22}$ G. Aeppli, S. M. Hayden, H. A. Mook, Z. Fisk, S. W. Cheong, D. Rytz, J. P. Remeika, G. P. Espinosa, and A. S. Cooper, Phys. Rev. Lett. 62, 2052 (1989).

${ }^{23}$ S. M. Hayden, G. Aeppli, R. Osborn, A. D. Taylon, T. G. Perring, S. W. Cheong, and Z. Fisk, Phys. Rev. Lett. 67, 3622 (1991).

${ }^{24}$ M. Greven, R. J. Birgeneau, Y. Endoh, M. A. Kastner, M. Matsuda, and G. Shirane, Z. Phys. B 96, 465 (1995).

${ }^{25}$ R. J. Cava, A. Santoro, D. W. Johnson, Jr., and W. W. Rhodes, Phys. Rev. B 35, 6716 (1987).

${ }^{26}$ L. L. Miller, X. L. Wang, S. X. Wang, C. Stassis, D. C. Johnston, J. Faber, Jr., and C.-K. Loong, Phys. Rev. B 41, 1921 (1990).

${ }^{27}$ C. J. Calzado, J. F. Sanz, and J.-P. Malrieu, J. Chem. Phys. 112, 5158 (2000).

${ }^{28}$ D. Muńoz, F. Illas, and I. de P. R. Moreira, Phys. Rev. Lett. 84, 1579 (2000).

${ }^{29}$ T. P. Mitchell, W. H. Bernard, and J. R. Wasson, Acta Crystallogr., Sect. B: Struct. Crystallogr. Cryst. Chem. 26, 2096 (1970).

${ }^{30}$ B. J. Cole and L. V. H. Brumage, J. Chem. Phys. 53, 4718 (1970).

${ }^{31}$ T. R. Felthouse, E. J. Laskowski, and D. N. Hendrickson, Inorg. Chem. 16, 1077 (1977)

${ }^{32}$ P. Chaudhuri, K. Oder, K. Wieghardt, B. Nuber, and J. Weiss, Inorg. Chem. 25, 2818 (1986).

${ }^{33}$ M. Julve, M. Verdaguer, A. Gleizes, M. Philoche-Levisalles, and O. Kahn, Inorg. Chem. 23, 3808 (1984).

${ }^{34}$ O. Castillo, I. Muga, A. Luque, J. M. Gutierrez-Zorrilla, J. Sertucha, P. Vitoria, and P. Roman, Polyhedron 18, 1235 (1999).

${ }^{35}$ Y. Journaux, J. Sletten, and O. Kahn, Inorg. Chem. 24, 4063 (1985).

${ }^{36}$ I. Castro, J. Faus, F. Lloret, M. Verdaguer, O. Kahn, S. Jeannin, Y. Jeannin, and J. Vaisserman, J. Chem. Soc. Dalton Trans. 1990, 2207 (1990).

${ }^{37}$ J. Cabrero, N. Ben Amor, C. de Graaf, F. Illas, and R. Caballol, J. Phys. Chem. A 104, 9983 (2000).

${ }^{38}$ P.-O. Widmark, B. J. Persson, and B. O. Roos, Theor. Chim. Acta 79, 419 (1991).

${ }^{39}$ K. Pierloot, B. Dumez, P.-O. Widmark, and B. O. Roos, Theor. Chim. Acta 90, 87 (1995).

${ }^{40}$ P.-O. Widmark, P.-Å. Malmqvist, and B. O. Roos, Theor. Chim. Acta 77, 291 (1990).

${ }^{41}$ CASDI suite of programs, D. Maynau. Laboratoire de Chimie et Physique Quantiques. Université de Toulouse.

${ }^{42}$ N. Ben Amor and D. Maynau, Chem. Phys. Lett. 286, 211 (1998).

${ }^{43}$ F. Aquilante, L. De Vico, N. Ferré, G. Ghigo, P.-Å. Malmqvist, P. Neogrády, T. B. Pedersen, M. Pitonak, M. Reiher, B. O. Roos, L. Serrano-Andrés, M. Urban, V. Veryazov, and R. Lindh, J. Comput. Chem. 31, 224 (2010).

${ }^{44}$ V. M. García, O. Castell, R. Caballol, and J.-P. Malrieu, Chem. Phys. Lett. 238, 222 (1995); D. Munoz, C. de Graaf, and F. Illas, J. Comput. Chem. 25, 1234 (2004).

${ }^{45}$ J. Cabrero, C. J. Calzado, D. Maynau, R. Caballol, and J.-P. Malrieu, J. Phys. Chem. A 106, 8146 (2002).

${ }^{46}$ B. Levy and G. Berthier, Int. J. Quantum Chem. 2, 307 (1968).

${ }^{47}$ C. Angeli, J. Comput. Chem. 30, 1319 (2009). 